\title{
Research of Self-learning of Johnson-Cook Models Parameters based on Genetic Algorithm
}

\author{
Chunyu He ${ }^{1, a}$, Zhijie Jiao ${ }^{1, b}$, Shaojie Wang ${ }^{1, \mathrm{c}}$, Fuxiang Zhao ${ }^{1, \mathrm{~d}}$ \\ ${ }^{1}$ State Key Lab of Rolling and Automation, Northeastern University, Shenyang 110819, China

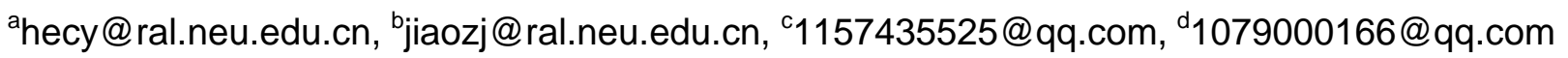

Keywords: plate, rolling, genetic algorithm, self-learning

Abstract. The Johnson-Cook models parameters of deformation resistance determine the prediction accuracy of rolling force during hot rolling. According to the influencing factors analysis of rolling force calculation error, the genetic algorithm was introduced into the self-learning method of Johnson-Cook models parameters, and searches the models optimal value on the basic of space exploration and optimization ability of genetic algorithm. The decision variable selection, the coding and decoding, the fitness evaluation and the terminal conditions process were implemented during development process of self-learning system. The results show that the optimization accuracy and speed can meet industrial production requirement.

\section{Introduction}

The Johnson-Cook model is an ideal rigid-plastic reinforcement model that reflects the strain-rate-hardening effect and temperature-softening [1-3]. Due to the clear meaning of its parameters, the Johnson-Cook model has been extensively used in numerical simulation software and rolling process calculations applications. However, because of alloying elements deviation and unstable rolling process parameters, there is often a large error between the calculated rolling force and the actual value. Therefore, in the production site complex factors, Johnson-Cook models parameters should be revised with the production of continuous learning in order to be able to provide accurate enough predictive value and the support for the high precision calculation of the on-line model during the hot rolling process[4,5].

In the artificial intelligence field, many issues need to be solved through finding the optimal solution or quasi-optimal solution from the complicated and enormous searching space. The researches on the general searching algorithm, which can automatically acquire and accumulate knowledge of searching space and adapt to control the searching process, thereby yielding optimal solution or quasi-optimal solution, has received wide attention. Genetic algorithm was generated in this context and has been proven to be particularly effective [6]. The corresponding relationship between actual detect data (including rolling force, rolling gap, temperature and so on) during production process and steel grade has been established. The space searching and optimizing abilities of genetic algorithm has been used to develop a deformation resistance self-learning system. Deformation resistance parameters of Johnson-Cook models are optimized in order to enhance on-line setting function.

\section{Calculation model models setup}

Johnson-Cook constitutive equation is empirical constitutive equation, Von Mises equivalent stress is the equivalent plastic strain, equivalent plastic strain rate and temperature functions, the Johnson-Cook equation is shown in Eq. (1).

$$
\sigma=\left(A+B \varepsilon^{n}\right)(1+C \ln \& \&)\left[1-T^{* m}\right]
$$


in it, $\mathrm{A}, \mathrm{B}, \mathrm{C}, \mathrm{m}, \mathrm{n}$ are models parameters; $\sigma$ is the Von Mises equivalent stress; $\varepsilon$ is equivalent plastic strain; \&\& $=\& \& \&$ \& is relative equivalent plastic strain rate, \&\& $=1.0 \mathrm{~s}^{-1} ; T^{*}=\left(T-T_{r}\right) /\left(T_{m}-T_{r}\right)$, $T_{m}$ is the melting point of the material, $T_{r}$ is is the ambient temperature.

In Eq. (1), A is the static yield stress at room temperature, which can be obtained experimentally. Therefore, we only need to optimize the parameters $\mathrm{B}, \mathrm{C}, \mathrm{m}, \mathrm{n}$ according to the production data.

The equation for calculating rolling force is often used SIMS model [7]. The SIMS model is given in Eq. (2).

$$
P=K \cdot Q_{\mathrm{P}} \cdot l_{\mathrm{C}}^{\prime} \cdot B
$$

where, $P$ is rolling force; $K$ is average deformation resistance; $Q_{\mathrm{P}}$ is yield stress state factor; $l_{\mathrm{C}}^{\prime}$ is the contact arc length with the working roll bruise considered; $B$ is plate width.

Whereas, plate width multiplied by the horizontal projected length of arc contact is the contact area. Contact area is the geometric determinant factor of rolling force; $Q_{\mathrm{P}}$ is mechanical determinant factor and $K$ is physical determinant factor. This model is widely agreed and applied by many manufactures. Sims rolling force model is one of the best solutions applied in production process. Deformation resistance is the most active factor, which is instead by the equivalent stress $\sigma$ of Johnson-Cook constitutive equation.

Plate rolling is a reversible rolling process. Exit thickness of the previous rolling pass will directly influence the next rolling pass. The predicted rolling force of each rolling pass needs to be compared with the actual rolling force in the optimization process of deformation resistance parameters. Therefore, precise exit thickness of each rolling pass is required. Spring model can be used to calculate the exit thickness of rolled piece [8,9]. The formula is given in Eq. (3).

$$
h=g a p+S+W-C+Z P C
$$

in it, gap is the average roll gap; $S$ is the mill elastic deformation; $W$ is the roll wear; $C$ is the thermal crown of the roll; $Z P C$ is the zero correction value.

\section{Self-learning system design}

Data generated in the production process are stored in the database in the designed format. Once the self-learning computer detects that the errors between prediction value and actual value of rolling force exceed the critical value, it will trigger deformation resistance self-learning process and thereby to optimize deformation resistance parameters. Historic rolling process data are required in this process. An appropriately designed data storage structure and easily access data source interface are required to facilitate deformation resistance parameters self-learning.

Self-leaning model of Johnson-Cook equation parameters includes genetic algorithm and rolling mechanism model. Procedures of self-learning are as follows:

(1) if the current rolling is completed, the rolling pass average data will be stored to database.

(2) to monitor whether the difference $\Delta P$ between actual rolling forces collected and predicted rolling force meet $|\Delta P|<\delta$. if met, trigger the self-learning computing;

(3) to extract data collected from ${ }^{n}$ recent rolling pieces from the database to be as a sample data;

(4) the deformation resistance parameter $\mathrm{B}, \mathrm{C}, \mathrm{m}, \mathrm{n}$ will be used as decision variables, the differences between predicted rolling force and actual rolling force of the same steel grade at different rolling passes will be the scale factor of fitness function. Then genetic algorithm model will be called to solve and optimize parameters;

(5) update parameters according to steel grades. 
In order to evaluate the similarity between average rolling force in pass and the predicted rolling force, the following objective function as Eq.(4) is chosen when deformation resistance has been optimized through genetic algorithm.

$$
f(X)=\left(\frac{\sum_{i=1}^{n}\left(P_{\text {avg }}^{i}-P_{c a l}^{i}\right)^{2}}{n}\right)^{0.5}
$$

in it, $n$ is the number of passes; $P_{a v g}^{i}$ is actual average rolling force on pass $i ; P_{c a l}^{i}$ is predicted rolling force on pass $i$.

The self-learning process of the Johnson-Cook eqution parameters can be taken as solving an optimization problem of $\min f(X)$.

\section{Production application}

One manufacturer wants to produce Q235B steel. The size of blank and product are $220 \mathrm{~mm} \times 2000 \mathrm{~mm} \times 3300 \mathrm{~mm}$ and $55 \mathrm{~mm} \times 2200 \mathrm{~mm}$ respectively, The parameter A of Johnson-Cook eqution is $249.4 \mathrm{MPa}$ at room temperature. The specifications of rolling and rolling pass data collected are illustrated in table 1.

Table 1 Pass average data

\begin{tabular}{clcccc}
\hline Pass & Status & $\begin{array}{c}\text { Setting gap } \\
(\mathrm{mm})\end{array}$ & $\begin{array}{c}\text { Mean temperature } \\
\left({ }^{\circ} \mathrm{C}\right)\end{array}$ & $\begin{array}{c}\text { Mean speed } \\
(\mathrm{r} / \mathrm{min})\end{array}$ & $\begin{array}{c}\text { Mean force } \\
(\mathrm{kN})\end{array}$ \\
\hline 1 & broadening & 212.36 & 1041 & 35 & 14400 \\
2 & broadening & 195.34 & 1037 & 35 & 22451 \\
3 & normal & 165.23 & 1023 & 35 & 25415 \\
4 & normal & 134.95 & 1013 & 40 & 29954 \\
5 & normal & 106.59 & 1009 & 40 & 30998 \\
6 & normal & 85.65 & 1004 & 40 & 26190 \\
7 & normal & 68.8 & 998 & 50 & 24516 \\
8 & normal & 55.8 & 991 & 50 & 22748 \\
\hline
\end{tabular}

Assume the parameter B, C, m, m in Eq. (1) which describes the Johnson-Cook eqution are decision variables and introduce the data to the rolling force model and genetic algorithm model. The initial values of parameters in the calculating process with genetic algorithm are as follows: (1) range of variable: $B \in(400,1000), C \in(0,1), m \in(0,1), n \in(0,2)$; (2) population size: 120 ; (3) selection operator: proportional selection; (4) crossover operator: one point crossover; (5) mutation operator: uniform mutation; (6) crossover probability: 0.8; (7) variation probability: 0.2.

During the process of selection, crossover and variation in population, the best solution is obtained in the 285th generation, where the fitness value becomes 359.85 and the parameters in self-learning process of the Johnson-Cook model parameters are as follows:

$$
B=843.8, C=0.106, m=0.758, \quad n=1.084
$$

These optimized parameters in self-learning process are introduced into the rolling force model and the comparison of actual average rolling force and predicted rolling force is shown in Figure 1.

The result shows that the average error between actual rolling force and the predicted force is only $318.4 \mathrm{kN}$ after using genetic algorithm to optimize the deformation parameters and introducing them to the model. Therefore, the high precision for Johnson-Cook parameters can be attained by using this parameter optimization method. 


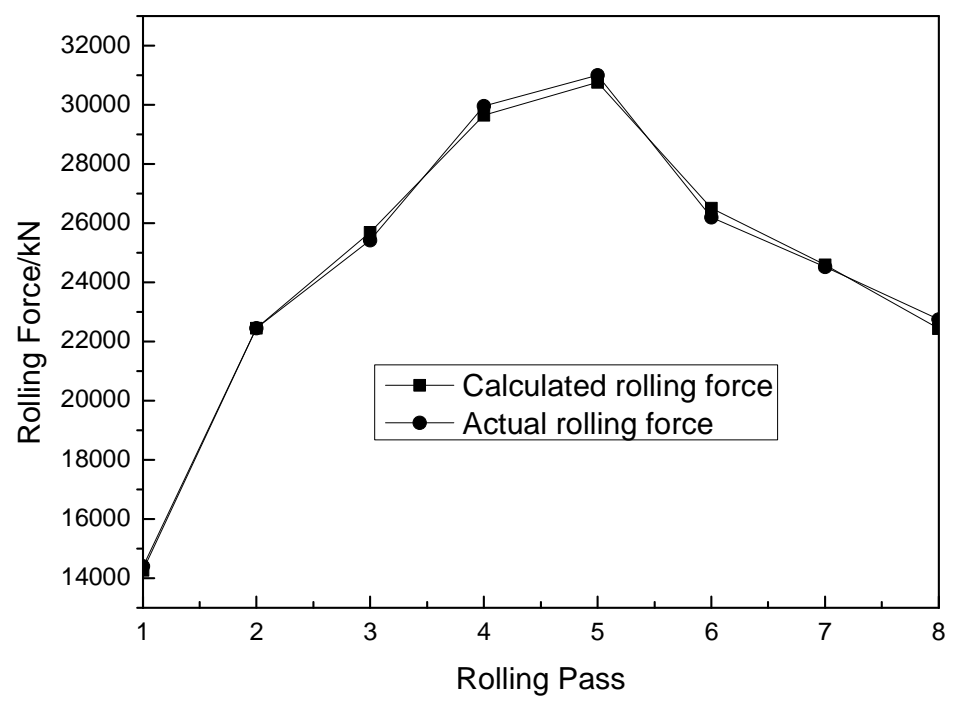

Fig.1 Comparison between actual and calculated rolling force

\section{Conclusion}

(1) The reason why there is large error using traditional model to predict the rolling force during the production of plates is analyzed. Combing with its biological basis, genetic algorithm is introduced into self-learning of Johnson-Cook parameters and then optimize these parameters.

(2) Through analysis of rolling force model, the procedures of genetic algorithm-based self-learning are proposed, and the requirements of developing a self-learning system of Johnson-Cook model parameters are identified. The selection of fitness evaluation function is also discussed in this paper.

(3) According to the production data, the self-learning system of deformation resistance based on genetic algorithm is introduced actual production. The results show that this method can optimize fast and produce precise outputs, which is fully met the production requirements of hot rolling.

\section{References}

[1] G.R. Johnson, W.H. Cook: Engineering Fracture Mechanics Vol. 21(1985), p. 31

[2] R.L. Matrineau, C.A. Anderson, F.W. Smith: EEExperimental Mechanics Vol. 40(2000), p. 219

[3] F.J. Zerilli, R.W. Armstrong: Jappl Phys Vol. 61(1987), p. 1816.

[4] A.C. Mackenzie, J.W. Hancock, D.K. Brown: Engng Fracture Mech Vol. 9(1977), p. 167

[5] Y. Bai, T. Wierzbicki: Int J Plast Vol. 24(2008), p. 1071

[6] S. Forrest, B. Javornik, R. Smith, A.S. Perelson: Evolutionary Computation Vol. 1(1993), p. 191

[7] R.B. Sims: Journal of the Iron and Steel Institute Vol. 168(1954), p. 6

[8] C.Y. He, D. Wu, X.M. Zhao: Journal of Northeastern University Vol. 30(2009), p. 1751

[9] X.L. Hu, G.S. Wu, S.G. Zhao: Steel Rolling Vol. 20(2003), p. 7 